\title{
Hyperbolic Relaxation of a Fourth Order Evolution Equation
}

\author{
Renato Colucci and Gerardo R. Chacón \\ Departamento de Matemáticas, Pontificia Universidad Javeriana, Cra. 7 No. 43-82, Bogotá, Colombia \\ Correspondence should be addressed to Renato Colucci; renatocolucci@hotmail.com
}

Received 26 November 2012; Revised 30 January 2013; Accepted 3 February 2013

Academic Editor: Juan J. Nieto

Copyright (c) 2013 R. Colucci and G. R. Chacón. This is an open access article distributed under the Creative Commons Attribution License, which permits unrestricted use, distribution, and reproduction in any medium, provided the original work is properly cited.

We propose a hyperbolic relaxation of a fourth order evolution equation, with an inertial term $\eta u_{t t}$, where $\eta \in(0,1]$. We prove the existence of several absorbing sets having different regularities and the existence of a global attractor that is bounded in $H^{4}(I) \times$ $\left\{H^{2}(I) \cap H_{0}^{1}(I)\right\}$

\section{Introduction}

Leting $I \subset \mathbb{R}$ be an open interval, with $|I| \leq 1$, we consider the following initial-boundary value problem for $u: I \times \mathbb{R}^{+} \rightarrow$ $\mathbb{R}$ :

$$
\begin{aligned}
\eta u_{t t}+u_{t}= & -\varepsilon^{2} u_{x x x x} \\
& +\frac{1}{2} W^{\prime \prime}\left(u_{x}\right) u_{x x}, \quad(x, t) \in I \times \mathbb{R}^{+}, \\
& u(x, 0)=u_{0}(x), \quad \text { in } I, \\
& u_{t}(x, 0)=u_{1}(x), \quad \text { in } I, \\
u & =u_{x x}=0, \quad \text { in } \partial I, t \geq 0,
\end{aligned}
$$

where the function $W(p)=\left(p^{2}-1\right)^{2}$ is the so-called doublewell potential, $0<\varepsilon \ll 1$ and $\eta \in(0,1]$ are positive parameters.

Problem (1), with $\eta=0$, was proposed in [1] where the global dynamics was studied. In particular, the dynamical behavior of the solutions for small values of the parameter $\varepsilon$ was studied by means of numerical experiments. The existence of three well-differentiated time scales with peculiar dynamical behavior was showen. In the first time scale of order $O\left(\varepsilon^{2}\right)$ there is the formation of microstructure (see [2]) in the region where the gradient of the initial datum falls in the nonconvex region of $W$; this phenomenon produces a drastic reduction of the energy of the initial datum. In the second time scale of order $O(1)$ the equation exhibits a heat equation-like behavior in the convex regions while slow motion in the nonconvex ones. In the last time scale of order $O\left(\varepsilon^{-2}\right)$ the equation shows a finite-dimensional behavior: the solution is approximately the union of consecutive segments and the dynamic is slow.

In [3], the third time scale was studied; the authors proved the existence of a global attractor $\mathscr{A}_{\varepsilon} \subset L^{2}(I)$ (see [4]) that is bounded in $H^{2}(I)$. The time for which the solutions enter the absorbing set $\mathscr{B}_{\varepsilon}$ is of order $O\left(\varepsilon^{-2}\right)$ and it is consistent with the estimates found in [1]. Moreover the authors proved the existence of an exponential attractor $\mathscr{I}_{\varepsilon}$ with finite fractal dimension of order $O\left(\varepsilon^{-10}\right)$. In [5] the authors proved the existence of an inertial manifold $\mathscr{M}_{\varepsilon}$ (see [6]) whose dimension is of order $O\left(\varepsilon^{-19}\right)$, and by the $n$ dimensional volume elements methods (see [7]) an estimate of the dimension of the global attractor of order $O\left(\varepsilon^{-1}\right)$ was found. This estimate is also consistent with the numerical experiments developed in [1]; in fact it was found that the wave length of the microstructure is of order $O\left(\varepsilon^{-1}\right)$.

In the last years the viscous and no viscous hyperbolic relaxation of the Cahn-Hilliard equation has been extensively investigated. The model was proposed in [8] while in [9] the existence of a family of exponential attractors was proved. The viscous and nonviscous perturbation has been studied in [10] where the existence of a family of global attractors that are upper semicontinuous with respect to the vanishing of perturbations parameters was proved. These results have been extended in 2 and 3 dimensions; see for example [11, 12] and the references therein.

Due to the similarity of problem (1) to the Cahn-Hilliard equation we consider it interesting to study the hyperbolic 
relaxation of the fourth order evolution equation proposed in [1]. In particular if $v$ is the solution of the Cahn-Hilliard equation

$$
v_{t}+\Delta\left[\varepsilon^{2} \Delta v-W^{\prime}(v)\right]=0,
$$

with Neumann boundary conditions:

$$
\frac{\partial}{\partial n} v=\frac{\partial}{\partial n} \Delta v=0, \quad x \in\{0,1\},
$$

then

$$
u(x)=\int_{0}^{x} v(s) d s
$$

is the solution of (1), with $\eta=0$, with the corresponding boundary conditions:

$$
u=u_{x x}=0, \quad x \in\{0,1\} .
$$

In the present work we put the problem in the correct mathematical framework and prove the existence of a global attractor $\mathscr{A}_{\eta, \varepsilon}$ while we have left the proof of the existence of exponential attractors for a forthcoming paper. In Sections 2 and 3 we define the solution semigroup in the appropriate spaces and present some important energy estimates. In Section 4 we prove the existence of several absorbing sets with different regularities while in the last section we prove the existence of the global attractor.

\section{Preliminaries}

We begin this section by defining the following Hilbert spaces that will be helpful for our analysis:

$$
\begin{gathered}
\mathscr{H}_{\eta, \varepsilon}=\left[H^{2}(I) \cap H_{0}^{1}(I)\right] \times L^{2}(I), \\
\mathscr{V}_{\eta, \varepsilon}=D(A) \times H^{2}(I),
\end{gathered}
$$

where

$$
D(A)=\left\{u \in H^{4}(I): u=u_{x x}=0 \text { in } \partial I\right\}
$$

is the domain of the differential operator $A=\partial^{4} / \partial x^{4}$. The above spaces are equipped with the norms

$$
\begin{gathered}
\|(u, v)\|_{\mathscr{H}_{\eta, \varepsilon}}^{2}=\frac{\varepsilon^{2}}{2}\left\|u_{x x}\right\|^{2}+\frac{\eta}{2}\|v\|^{2}, \\
\|(u, v)\|_{\mathscr{V}_{\eta, \varepsilon}}^{2}=\frac{\varepsilon^{2}}{2}\left\|u_{\mathrm{x} x x x}\right\|^{2}+\frac{\eta}{2}\left\|v_{x x}\right\|^{2},
\end{gathered}
$$

where $\|\cdot\|$ represents the $L^{2}$ norm. We will denote by $\langle\cdot, \cdot\rangle$ the inner product in $L^{2}(I)$. We recall that for all $u \in H^{2}(I) \cap H_{0}^{1}(I)$ with $|I| \leq 1$, we have

$$
\|u\| \leq\left\|u_{x}\right\| \leq\left\|u_{x x}\right\| .
$$

Throughout the paper we will use two norms that are equivalent to (9) and (10) in order to simplify the computation.
Given functions $u \in H^{2}(I) \cap H_{0}^{1}(I)$ and $v \in L^{2}(I)$ we define the function $\widetilde{E}$ on $\mathscr{H}_{\eta, \varepsilon}$ by

$$
\widetilde{E}(u, v)=\eta\|v\|^{2}+\frac{1}{2} \varepsilon^{2}\left\|u_{x x}\right\|^{2}+\beta \eta\langle v, u\rangle,
$$

where $\beta \in\left[0, \varepsilon^{2}\right]$.

Proposition 1. For all $\beta \in\left[0, \varepsilon^{2}\right]$ the function $\widetilde{E}(\cdot, \cdot)$ induces a norm equivalent to the norm on $\mathscr{H}_{\eta, \varepsilon}$.

Proof. By Schwartz inequality (11) and using the fact that $\beta \leq$ $\varepsilon^{2}<1, \eta \in(0,1]$, we get

$$
\begin{aligned}
\beta \eta\langle v, u\rangle & \leq \beta \eta\|v\|\|u\| \leq \frac{1}{2} \eta^{2}\|v\|^{2}+\frac{1}{2} \varepsilon^{4}\left\|u_{x x}\right\|^{2} \\
& \leq \frac{\eta}{2}\|v\|^{2}+\frac{\varepsilon^{2}}{2}\left\|u_{x x}\right\|^{2}=\|(u, v)\|_{\mathscr{H}_{\varepsilon, \eta}}^{2}
\end{aligned}
$$

From the previous inequality and by definition of $\widetilde{E}(u, v)$ we get

$$
\widetilde{E}(u, v) \leq 3\|(u, v)\|_{\mathscr{H}}^{2} .
$$

By a different application of Schwartz inequality and from (11) we get

$$
\begin{aligned}
\beta \eta\langle v, u\rangle & \leq \beta \eta\|v\|\|u\| \leq \eta^{2}\|v\|^{2}+\frac{\beta^{2}}{4}\|u\|^{2} \\
& \leq \eta\|v\|^{2}+\frac{\varepsilon^{4}}{4}\|u\|^{2} \leq \eta\|v\|^{2}+\frac{\varepsilon^{2}}{4}\left\|u_{x x}\right\|^{2} .
\end{aligned}
$$

Combining (13) and (15) we get

$$
\widetilde{E}(u, v) \geq \max \left\{\frac{\eta}{2}\|v\|^{2}, \frac{1}{4} \varepsilon^{2}\left\|u_{x x}\right\|^{2}\right\},
$$

and as a consequence

$$
\widetilde{E}(u, v) \geq \frac{1}{3}\|(u, v)\|_{\mathscr{H}_{\varepsilon, \eta}}^{2} .
$$

The proof of the following theorem follows from classical applications of the Faedo-Galerkin method. We will only show a Lipschitz estimate that will be needed for further computations.

Theorem 2. For every $\left(u_{0}, u_{1}\right) \in \mathscr{H}_{\varepsilon, \eta}$ there exists a unique solution $u(t)$ for the initial value problem (1) such that

$$
u \in C_{b}\left(\mathbb{R}^{+} ; H^{2}(I) \cap H_{0}^{1}(I)\right) \cap C_{b}^{1}\left(\mathbb{R}^{+} ; L^{2}(I)\right) .
$$

If, moreover, $\left(u_{0}, u_{1}\right) \in \mathscr{V}_{\varepsilon, \eta}$, then:

$$
\begin{gathered}
u \in C_{b}\left(\mathbb{R}^{+} ; D(A)\right) \cap C_{b}^{1}\left(\mathbb{R}^{+} ; H^{2}(I) \cap H_{0}^{1}(I)\right) \\
\cap C_{b}^{2}\left(\mathbb{R}^{+} ; L^{2}(I)\right) .
\end{gathered}
$$


Proposition 3. For any constant $R \geq 0$ there exists a positive constant $K=K(R)$ such that, for any initial data $u_{1}(0), u_{2}(0)$ with $\left\|u_{i}(0)\right\|_{H_{\eta, \varepsilon}} \leq R, i=1,2$ one has

$$
\left\|S_{\varepsilon, \eta}(t) u_{1}(0)-S_{\varepsilon, \eta}(t) u_{2}(0)\right\|_{H_{\eta, \varepsilon}} \leq e^{\left(K^{2} / \varepsilon^{2}\right) t}\left\|u_{1}(0)-u_{2}(0)\right\|_{H_{\eta, \varepsilon}},
$$

where $S_{\varepsilon, \eta}(t)$ is the solution semigroup of the problem (1).

Proof. Let $u_{1}, u_{2}$, two solutions of (1) with initial data $u_{1}(0)$ and $u_{2}(0)$. Let, $w=u_{1}-u_{2}$ then we write

$$
\eta w_{t t}+w_{t}+\varepsilon^{2} w_{x x x x}=\frac{1}{2} \frac{d}{d x}\left\{W^{\prime}\left(u_{1 x}\right)-W^{\prime}\left(u_{2 x}\right)\right\}
$$

We multiply the above equation by $w_{t}$ in $L^{2}(I)$; then

$$
\begin{aligned}
\frac{\eta}{2} \frac{d}{d t}\left\|w_{t}\right\|^{2}+\frac{\varepsilon^{2}}{2} \frac{d}{d t}\left\|w_{x x}\right\|^{2}+\left\|w_{t}\right\|^{2} \\
=-2 \int_{I} w_{x x} w_{t} d x \\
+6 \int_{I}\left\{u_{1 x}^{2} w_{x x}+u_{2 x x} w_{x}\left[u_{1 x}+u_{2 x}\right]\right\} w_{t} d x \\
\leq 2\left\|w_{x x}\right\|\left\|w_{t}\right\|+6\left\|u_{1 x}\right\|_{\infty}^{2}\left\|w_{x x}\right\|\left\|w_{t}\right\| \\
+12\left\|u_{2 x x}\right\|\left(\int_{I} w_{x}^{2} w_{t}^{2}\left[u_{1 x}^{2}+u_{2 x}^{2}\right] d x\right)^{1 / 2} \\
\leq 2\left\{1+18 C^{2}\left\|u_{1 x x}\right\|^{2}+12\left\|u_{2 x x}\right\|\right. \\
\left.\times\left(\left\|u_{1 x x}\right\|^{2}+\left\|u_{2 x x}\right\|^{2}\right)^{1 / 2}\right\}\left\|w_{x x}\right\|\left\|w_{t}\right\| \\
\leq 2\left\{1+\left(36 C^{2}+24 \sqrt{2}\right) \frac{R^{2}}{\varepsilon^{2}}\right\}\left\|w_{x x}\right\|\left\|w_{t}\right\| \\
\leq 2 K\left\|w_{x x}\right\|\left\|w_{t}\right\| \leq K^{2}\left\|w_{x x}\right\|^{2}+\left\|w_{t}\right\|^{2},
\end{aligned}
$$

where we have used the following inequality (see [13] and inequality (11)):

$$
\left\|u_{x}\right\|_{\infty}^{2} \leq C^{2}\left\|u_{x}\right\|_{H^{1}(I)}^{2} \leq 2 C^{2}\left\|u_{x x}\right\|^{2}
$$

The constant $K$ can be explicitly computed using the estimates of the absorbing set presented in the next section.

From the last inequality we get

$$
\frac{d}{d t}\left\|\left(w, w_{t}\right)\right\|_{\mathscr{H}_{\varepsilon, \eta}}^{2} \leq \frac{2 K^{2}}{\varepsilon^{2}}\left\|\left(w, w_{t}\right)\right\|_{\mathscr{H}_{\varepsilon, \eta}}^{2},
$$

and then using Gronwall's lemma we get

$$
\left\|\left(w, w_{t}\right)\right\|_{\mathscr{H}_{\varepsilon, \eta}} \leq e^{\left(K^{2} / \varepsilon^{2}\right) t}\left\|\left(w(0), w_{t}(0)\right)\right\|_{\mathscr{H}_{\varepsilon, \eta}} .
$$

\section{A Priori Estimates}

In this section we provide useful a priori estimates of energy type. Equation (1) admits a Liapunov functional of the form:

$$
E\left(u, u_{t}\right)=\eta\left\|u_{t}\right\|^{2}+\frac{1}{2} \varepsilon^{2}\left\|u_{x x}\right\|^{2}+\frac{1}{2} \int_{I} W\left(u_{x}\right) d x,
$$

that is not increasing along the solutions; in fact if we multiply (1) by $u_{t}$ and integrate over $I$ we obtain

$$
\frac{d}{d t} E\left(u, u_{t}\right)=-\left\|u_{t}\right\|^{2}
$$

Moreover, integrating the previous inequality on $(0, t)$ with respect to time we get

$$
\int_{0}^{t}\left\|u_{t}\right\|^{2} d s+E\left(u, u_{t}\right)=E\left(u_{0}, u_{1}\right)
$$

Then for any fixed initial data $\left(u_{0}, u_{1}\right) \in \mathscr{H}_{\varepsilon, \eta}$ we have that the corresponding solution satisfies

$$
\begin{aligned}
\sup _{t \geq 0}\left\{\frac{\eta}{2}\left\|u_{t}\right\|^{2}+\frac{\varepsilon^{2}}{2}\left\|u_{x x}\right\|^{2}\right\} & =\sup _{t \geq 0}\left\|\left(u, u_{t}\right)\right\|_{\mathscr{H}_{\varepsilon, \eta}}^{2} \\
& \leq E\left(u_{0}, u_{1}\right) .
\end{aligned}
$$

Moreover if we integrate over $\mathbb{R}^{+}$we get the integral control

$$
\int_{0}^{\infty}\left\|u_{t}\right\|^{2} d t \leq E\left(u_{0}, u_{1}\right)
$$

We consider an important estimate that will be useful later. Let $\beta>0$ be a parameter to be determined later; if we multiply (1) by $\beta u$ we obtain

$$
\begin{gathered}
\beta \eta \int_{I} u_{t t} u d x+\frac{\beta}{2} \frac{d}{d t}\|u\|^{2}+\beta \varepsilon^{2}\left\|u_{x x}\right\|^{2} \\
+2 \beta\left\|u_{x}\right\|_{4}^{4}=2 \beta\left\|u_{x}\right\|^{2} .
\end{gathered}
$$

By summing (27) and (31) we get

$$
\begin{aligned}
\frac{d}{d t} E+ & \beta \eta \frac{d}{d t}\left\langle u_{t}, u\right\rangle+(1-\beta \eta)\left\|u_{t}\right\|^{2} \\
& +\frac{\beta}{2} \frac{d}{d t}\|u\|^{2}+\beta \varepsilon^{2}\left\|u_{x x}\right\|^{2}+2 \beta\left\|u_{x}\right\|_{4}^{4} \\
= & 2 \beta\left\|u_{x}\right\|^{2} .
\end{aligned}
$$

Using the expression of the energy we can rewrite the previous in the following way:

$$
\begin{aligned}
\frac{d}{d t} E+ & \beta \eta \frac{d}{d t}\left\langle u_{t}, u\right\rangle+(1-3 \beta \eta)\left\|u_{t}\right\|^{2} \\
& +\beta\left\langle u_{t}, u\right\rangle+2 \beta\left(E+\frac{\left\|u_{x}\right\|_{4}^{4}}{2}-\frac{|I|}{2}\right)=0 .
\end{aligned}
$$

We will estimate some of the terms of the previous inequality in the following lemma. 
Lemma 4. Fix $\eta \leq 1$ then for all $\beta \in(0,1 / 3)$ one has

$$
2 \beta^{2} \eta\left\langle u_{t}, u\right\rangle \leq(1-3 \beta \eta)\left\|u_{t}\right\|^{2}+\beta\left\|u_{x}\right\|_{4}^{4}+\beta|I|+\beta\left\langle u_{t}, u\right\rangle .
$$

Proof. By Holder's inequality we get

$$
2\left\|u_{x}\right\|^{2} \leq 2\left\|u_{x}\right\|_{4}^{2}|I|^{1 / 2} \leq\left\|u_{x}\right\|_{4}^{4}+|I|,
$$

then we use Poincarés inequality and the fact that $|I| \leq 1$ to conclude that

$$
\|u\|^{2} \leq \frac{\left\|u_{x}\right\|_{4}^{4}}{2}+\frac{|I|}{2} .
$$

Therefore, for a positive constant $c_{1}$ to be determined later, we have that

$$
\begin{aligned}
\left\langle u_{t}, u\right\rangle & \leq\left\|u_{t}\right\|\|u\| \leq \frac{c_{1}}{2}\left\|u_{t}\right\|^{2}+\frac{1}{2 c_{1}}\|u\|^{2} \\
& \leq \frac{c_{1}}{2}\left\|u_{t}\right\|^{2}+\frac{1}{2 c_{1}}\left(\frac{\left\|u_{x}\right\|_{4}^{4}}{2}+\frac{|I|}{2}\right) .
\end{aligned}
$$

Consequently, by choosing the parameters $c_{1}=1 / 12$ and $\beta<$ $1 / 3$ we have that

$$
\begin{aligned}
& \left(2 \beta^{2} \eta-\beta\right)\left\langle u_{t}, u\right\rangle \\
& \leq\left|2 \beta^{2} \eta-\beta\right| \frac{c_{1}}{2}\left\|u_{t}\right\|^{2} \\
& \quad+\frac{\left|2 \beta^{2} \eta-\beta\right|}{4 c_{1}}\left\|u_{x}\right\|_{4}^{4}+\frac{\left|2 \beta^{2} \eta-\beta\right|}{4 c_{1}}|I| \\
& \leq(1-3 \beta \eta)\left\|u_{t}\right\|^{2}+\beta\left\|u_{x}\right\|_{4}^{4}+\beta|I| .
\end{aligned}
$$

Now, using the previous lemma and (33) we conclude that

$$
\frac{d}{d t} E+\beta \eta \frac{d}{d t}\left\langle u_{t}, u\right\rangle+2 \beta^{2} \eta\left\langle u_{t}, u\right\rangle+2 \beta E \leq 2 \beta|I| .
$$

If we set

$$
\Phi\left(u, u_{t}\right)=\Phi(t)=E\left(u, u_{t}\right)+\beta \eta\left\langle u_{t}, u\right\rangle
$$

then from (39) we get

$$
\frac{d}{d t} \Phi(t)+2 \beta \Phi(t) \leq 2 \beta \text {. }
$$

Now, integrating we have that

$$
\Phi(t) \leq\left[\Phi\left(u_{0}, u_{1}\right)-1\right] e^{-2 \beta t}+1 .
$$

The previous inequality will be used in the next section to show the existence of absorbing sets for the problem (1).

We remark that the following inequality holds for the functions $E, \widetilde{E}$, and $\Phi$ :

$$
\Phi(t)=\widetilde{E}(t)+\frac{1}{2} \int_{I} W\left(u_{x}\right) d x \geq \widetilde{E} \geq 0 .
$$

\section{Absorbing Sets}

In this section we will show the existence of several absorbing sets for the solution semigroup of (1) in the space $\mathscr{H}_{\varepsilon, \eta}$. Also, assuming further regularity of the initial data, the existence of a more regular absorbing sets is also shown.

First, notice the following estimate of the nonlinear term of the energy functional defined in (26). Using (23) we get

$$
\begin{aligned}
\frac{1}{2} \int_{I} W\left(u_{x}\right) d x & \leq \frac{1}{2}\left\|u_{x}\right\|^{2}\left\|u_{x}\right\|_{L^{\infty}(I)}^{2}-\left\|u_{x}\right\|^{2}+\frac{|I|}{2} \\
& \leq \frac{1}{2}\left\|u_{x}\right\|^{2}\left\|u_{x x}\right\|^{2}+\frac{|I|}{2} \\
& \leq \frac{1}{2}\left\|u_{x x}\right\|^{4}+\frac{|I|}{2} .
\end{aligned}
$$

We will use this inequality several times in what follows.

\subsection{Absorbing Set in $\mathscr{H}_{\varepsilon, \eta}$}

Proposition 5. For all $R_{0}>1$ the set

$$
B_{\Phi}:=\left\{\left(u, u_{t}\right) \in \mathscr{H}_{\varepsilon, \eta}: \Phi\left(u, u_{t}\right) \leq R_{0}\right\}
$$

is bounded, absorbing, and positively invariant for the semigroup $S_{\varepsilon, \eta}(t)$ in $\mathscr{H}_{\varepsilon, \eta}$.

Proof. The set is positively invariant, in fact if $\left(u_{0}, u_{1}\right) \in \mathscr{H}_{\varepsilon, \eta}$ with $\Phi\left(u_{0}, u_{1}\right) \leq R_{0}$ we have, from (42), that

$$
\Phi\left(u, u_{t}\right) \leq\left[R_{0}-1\right] e^{-2 \beta t}+1 \leq R_{0}, \quad \forall t>0 .
$$

The boundness of $B_{\Phi}$ follows directly from (42). Now suppose that $\left(u_{0}, u_{1}\right)$ are such that $\Phi\left(u_{0}, u_{1}\right) \leq R$, with $R>R_{0}$ then again from (42) we get

$$
\Phi\left(u, u_{t}\right) \leq R_{0}, \quad \forall t \geq \tau_{\Phi},
$$

where

$$
\tau_{\Phi}:=\frac{1}{2 \beta} \log \left\{\frac{R-1}{R_{0}-1}\right\} .
$$

Then $B_{\Phi}$ is absorbing for the semigroup $S_{\varepsilon, \eta}(t)$.

Proposition 6. For all $R_{1}>1$ one has that the set

$$
B_{\widetilde{E}}:=\left\{\left(u, u_{t}\right) \in \mathscr{H}_{\varepsilon, \eta}: \widetilde{E}\left(u, u_{t}\right) \leq R_{1}\right\}
$$

is absorbing for the semigroup $S_{\varepsilon, \eta}(t)$.

Proof. We have from (42) and (43) that

$$
\widetilde{E}\left(u, u_{t}\right) \leq\left[\Phi\left(u_{0}, u_{1}\right)-1\right] e^{-2 \beta t}+1 .
$$

Now, take $\left(u_{0}, u_{1}\right)$ such that $\widetilde{E}\left(u_{0}, u_{1}\right) \leq R$, with $R>R_{1}$. Then, by (42) and (44) we get that

$$
\begin{aligned}
\widetilde{E}(t) & \leq \Phi(t) \leq \widetilde{E}(t)+\frac{1}{2}\left\|u_{x}\right\|_{4}^{4}+\frac{|I|}{2} \\
& \leq \widetilde{E}(t)+\frac{1}{2}\left\|u_{x x}\right\|^{4}+\frac{|I|}{2} \\
& \leq \widetilde{E}(t)+\frac{4}{\varepsilon^{2}} \widetilde{E}(t)^{2}+\frac{|I|}{2} .
\end{aligned}
$$


Therefore,

$$
\begin{aligned}
\widetilde{E}\left(u, u_{t}\right) & \leq\left\{\widetilde{E}\left(u_{0}, u_{1}\right)+\frac{4}{\varepsilon^{2}} \widetilde{E}\left(u_{0}, u_{1}\right)^{2}+\frac{|I|}{2}-1\right\} e^{-2 \beta t}+1 \\
& \leq\left\{R+\frac{4}{\varepsilon^{2}} R^{2}+\frac{|I|}{2}-1\right\} e^{-2 \beta t}+1
\end{aligned}
$$

Consequently, we have that

$$
\widetilde{E}\left(u, u_{t}\right) \leq R_{1}, \quad \forall t \geq \tau_{\widetilde{E}}
$$

where

$$
\tau_{\widetilde{E}}=\frac{1}{2 \beta} \log \left\{\frac{R+\left(4 / \varepsilon^{4}\right) R^{2}+(|I| / 2)-1}{R_{1}-1}\right\} .
$$

Now using the equivalence of the norm on $\mathscr{H}_{\varepsilon, \eta}$ and (52) we get

$$
\begin{aligned}
\left\|\left(u, u_{t}\right)\right\|_{\mathscr{H}_{\varepsilon, \eta}}^{2} \leq 3\{3 & \left\|\left(u_{0}, u_{1}\right)\right\|_{\mathscr{H}_{\varepsilon, \eta}}^{2} \\
& \left.+\frac{36}{\varepsilon^{2}}\left\|\left(u_{0}, u_{1}\right)\right\|_{\mathscr{H}_{\varepsilon, \eta}}^{4}+\frac{|I|}{2}-1\right\} e^{-2 \beta t}+3 .
\end{aligned}
$$

Let $R_{2}>\sqrt{3}$. If $\left(u_{0}, u_{1}\right) \in \mathscr{H}_{\varepsilon, \eta}$ is such that

$$
\left\|\left(u_{0}, u_{1}\right)\right\|_{\mathscr{H}_{\varepsilon, \eta}} \leq R
$$

then we have that

$$
\left\|\left(u, u_{t}\right)\right\|_{\mathscr{H}_{\varepsilon, \eta}} \leq R_{2}, \quad \forall t \geq \tau_{H}
$$

where

$$
\tau_{H}=\frac{1}{2 \beta} \log \frac{3\left\{3 R^{2}+\left(36 / \varepsilon^{2}\right) R^{4}+|I| / 2-1\right\}}{R_{2}^{2}-3} .
$$

Thus, we have proved the following.

Proposition 7. For all $R_{2}>\sqrt{3}$ one has that the ball

$$
B_{\mathscr{H}_{\varepsilon, \eta}}:=\left\{\left(u, u_{t}\right) \in \mathscr{H}_{\varepsilon, \eta}:\left\|\left(u, u_{t}\right)\right\|_{\mathscr{H}_{\varepsilon, \eta}} \leq R_{2}\right\}
$$

is an absorbing set for the semigroup $S_{\varepsilon, \eta}(t)$ in $\mathscr{H}_{\varepsilon, \eta}$.

4.2. Absorbing Set in $\mathcal{U}_{\varepsilon, \eta}$. Now suppose that the initial data has some additional regularity. Then we can prove the existence of more regular absorbing sets. Let us define the following space:

$$
\mathscr{U}_{\varepsilon, \eta}=\left\{H^{3}(I) \cap H^{2}(I) \cap H_{0}^{1}(I)\right\} \times H^{1}(I),
$$

equipped with the norm

$$
\left\|\left(u, u_{t}\right)\right\|_{u_{\varepsilon, \eta}}^{2}=\frac{\eta}{2}\left\|u_{t x}\right\|^{2}+\frac{\varepsilon^{2}}{2}\left\|u_{x x x}\right\|^{2} .
$$

Then we have the following.
Proposition 8. There exists $R_{3}>0$ such that the closed ball

$$
\mathscr{B}_{3}=\left\{\left(u, u_{t}\right) \in \mathscr{U}_{\varepsilon, \eta}:\left\|\left(u, u_{t}\right)\right\|_{\mathcal{U}_{\varepsilon, \eta}} \leq R_{3}\right\},
$$

is a bounded absorbing set for $S_{\varepsilon, \eta}(t)$ in $\mathcal{U}_{\varepsilon, \eta}$.

Proof. If we multiply (1) by $u_{x x t}+u_{x x}$ and integrate over $I$ we obtain

$$
\begin{aligned}
\frac{d}{d t}\left\{\frac{\eta}{2}\left\|u_{t x}\right\|^{2}+\frac{\varepsilon^{2}}{2}\left\|u_{x x x}\right\|^{2}+\frac{1}{2}\left\|u_{x}\right\|^{2}+\eta\left\langle u_{t x}, u_{x}\right\rangle\right\} \\
+(1-\eta)\left\|u_{t x}\right\|^{2}+\varepsilon^{2}\left\|u_{x x x}\right\|^{2} \\
=-\frac{1}{2}\left\langle\left[W^{\prime}\left(u_{x}\right)\right]_{x}, u_{x x t}\right\rangle-\frac{1}{2}\left\langle\left[W^{\prime}\left(u_{x}\right)\right]_{x}, u_{x x}\right\rangle .
\end{aligned}
$$

Let us denote the differential term of the previous inequality as

$$
\begin{aligned}
\Psi_{0}(t)= & \frac{\eta}{2}\left\|u_{t x}\right\|^{2}+\frac{\varepsilon^{2}}{2}\left\|u_{x x x}\right\|^{2}+\eta\left\langle u_{t x}, u_{x}\right\rangle \\
& +\frac{1}{2}\left\|u_{x}\right\|^{2} .
\end{aligned}
$$

We will estimate the right hand side of (63). The first term can be estimated as follows:

$$
\begin{aligned}
-\frac{1}{2}\left\langle\left[W^{\prime}\left(u_{x}\right)\right]_{x}, u_{x x}\right\rangle & \leq \frac{1}{2}\left\|W^{\prime \prime}\left(u_{x}\right)\right\|_{\infty}\left\|u_{x x}\right\|^{2} \\
& \leq 2\left(3\left\|u_{x}\right\|_{\infty}^{2}+1\right)\left\|u_{x x}\right\|^{2} \\
& \leq 2\left(3\left\|u_{x x}\right\|^{2}+1\right)\left\|u_{x x}\right\|^{2} \\
& \leq \frac{4}{\varepsilon^{2}} R_{2}^{2}\left(\frac{6 R_{2}^{2}}{\varepsilon^{2}}+1\right):=C_{1} .
\end{aligned}
$$

Let us define

$$
\Psi_{1}=\frac{1}{4} \int_{I} W^{\prime \prime}\left(u_{x}\right) u_{x x}^{2} d x
$$

then we can rewrite the the second term of r.h.s of (63)

$$
-\frac{1}{2}\left\langle\left[W^{\prime}\left(u_{x}\right)\right]_{x}, u_{x x t}\right\rangle=-\frac{d}{d t} \Psi_{1}+\frac{1}{4} \int_{I} W^{\prime \prime \prime}\left(u_{x}\right) u_{x t} u_{x x}^{2}
$$

and estimate 


$$
\begin{aligned}
\frac{1}{4} \int_{I} & W^{\prime \prime \prime}\left(u_{x}\right) u_{x t} u_{x x}^{2} \\
& \leq 6\left\|u_{x x}\right\|_{\infty} \int_{I} u_{x} u_{x t} u_{x x} d x \\
& \leq 6\left\|u_{x x}\right\|_{\infty}\left\|u_{x t}\right\|\left\|u_{x} u_{x x}\right\| \\
& \leq 6\left\|u_{x x}\right\|_{\infty}\left\|u_{x t}\right\|\left\|u_{x x}\right\|^{2} \\
& \leq 6\left\|u_{x x}\right\|^{5 / 2}\left\|u_{x x x}\right\|^{1 / 2}\left\|u_{x t}\right\| \\
& \leq 6\left(\frac{\sqrt{2}}{\varepsilon} R_{2}\right)^{5 / 2}\left\|u_{x x x}\right\|^{1 / 2}\left\|u_{x t}\right\|^{5}\left\|u_{x x x}\right\| \\
& \left.\leq \frac{\eta}{2}\left\|u_{x t}\right\|^{2}+\frac{18}{\eta}\left(\frac{\sqrt{2}}{\varepsilon} R_{2}\right)^{5}\right]^{2}+\frac{\varepsilon^{2}}{2}\left\|u_{x x x}\right\|^{2} \\
& \leq \frac{\eta}{2}\left\|u_{x t}\right\|^{2}+\frac{1}{2}\left[\frac{18}{\eta \varepsilon}\left(\frac{\sqrt{2}}{\varepsilon} R_{2}\right)^{5} \|^{2}+C_{2} .\right. \\
& :=\frac{\eta}{2}\left\|u_{x t}\right\|^{2}+\frac{\varepsilon^{2}}{2}\left\|u_{x x x}\right\|^{2}
\end{aligned}
$$

Then if $\eta \in(0,1 / 2)$ and setting $\Psi:=\Psi_{0}+\Psi_{1}, C:=C_{1}+C_{2}$ we have

$$
\frac{d}{d t} \Psi+\frac{\eta}{2}\left\|u_{x t}\right\|^{2}+\frac{\varepsilon^{2}}{2}\left\|u_{x x x}\right\|^{2} \leq C .
$$

To conclude the proof we note that

$$
\begin{aligned}
\Psi \geq & \left\|\left(u, u_{t}\right)\right\|_{u_{\varepsilon, \eta}}^{2}+\frac{1}{2}\left\|u_{x x}\right\|^{2} \\
& -\eta\left\|u_{t x}\right\|\left\|u_{x}\right\|-\left\|u_{x x}\right\|^{2} \\
\geq & \left\|\left(u, u_{t}\right)\right\|_{\mathscr{U}_{\varepsilon, \eta}}^{2}-\frac{\eta}{4}\left\|u_{t x}\right\|^{2}-\frac{3}{2}\left\|u_{x x}\right\|^{2} \\
\geq & \frac{1}{2}\left\|\left(u, u_{t}\right)\right\|_{\mathscr{U}_{\varepsilon, \eta}}^{2}-\frac{3}{\varepsilon^{2}} R_{2}^{2} \\
:= & \frac{1}{2}\left\|\left(u, u_{t}\right)\right\|_{\mathcal{u}_{\varepsilon, \eta}}^{2}-c_{1},
\end{aligned}
$$

and that

$$
\begin{aligned}
\Psi \leq & \left\|\left(u, u_{t}\right)\right\|_{u_{\varepsilon, \eta}}^{2}+\frac{\eta}{2}\left\|u_{t x}\right\|^{2}+\left\|u_{x x}\right\|^{2} \\
& +\left\|u_{x x}\right\|^{2}\left(3\left\|u_{x}\right\|_{\infty}^{2}+1\right) \\
\leq & 2\left\|\left(u, u_{t}\right)\right\|_{u_{\varepsilon, \eta}}^{2}+\frac{2}{\varepsilon^{2}} R_{2}^{2}\left(\frac{6}{\varepsilon^{2}} R_{2}^{2}+2\right) \\
:= & 2\left\|\left(u, u_{t}\right)\right\|_{u_{\varepsilon, \eta}}^{2}+c_{2} .
\end{aligned}
$$

From the previous inequalities we get

$$
\frac{1}{2}\left\|\left(u, u_{t}\right)\right\|_{\mathscr{U}_{\varepsilon, \eta}}^{2}-c_{1} \leq \Psi \leq 2\left\|\left(u, u_{t}\right)\right\|_{\mathcal{u}_{\varepsilon, \eta}}^{2}+c_{2} .
$$

Then from (72) we get

$$
\frac{d}{d t} \Psi+\frac{1}{2} \Psi \leq C+c_{2}:=\widetilde{C}
$$

and by Gronwall's lemma we obtain

$$
\begin{gathered}
\Psi(t) \leq(\Psi(0)-2 \widetilde{C}) e^{-(1 / 2) t}+2 \widetilde{C} \\
\left\|\left(u, u_{t}\right)\right\|_{\mathscr{U}_{\varepsilon, \eta}}^{2} \leq \\
+4 \widetilde{C}+2 c_{1} .
\end{gathered}
$$

Then if $\left(u_{0}, u_{1}\right) \in \mathcal{U}_{\varepsilon, \eta}$ such that

$$
\left\|\left(u_{0}, u_{1}\right)\right\|_{u_{\varepsilon, \eta}} \leq R
$$

we have that

$$
\left\|\left(u, u_{t}\right)\right\|_{u_{\varepsilon, \eta}} \leq R_{3}, \quad \forall t \geq \tau_{U}
$$

where

$$
\tau_{U}:=2 \log \left(\frac{2 R^{2}+2 c_{2}-4 \widetilde{C}}{R_{3}^{2}-4 \widetilde{C}-2 c_{1}}\right)
$$

and this concludes the proof.

4.3. Absorbing Set in $\mathscr{V}_{\varepsilon, \eta}$. We consider the space $\mathscr{V}_{\varepsilon, \eta}$ as defined in (7), equipped with the norm defined in (10). By considering more regular initial conditions we can prove the following result.

Proposition 9. There exists $R_{4}>0$ such that the closed ball

$$
\mathscr{B}_{4}=\left\{\left(u, u_{t}\right) \in \mathscr{V}_{\varepsilon, \eta}:\left\|\left(u, u_{t}\right)\right\|_{\mathscr{V}_{\varepsilon, \eta}} \leq R_{4}\right\}
$$

is a bounded absorbing set for the semigroup $S_{\varepsilon, \eta}(t)$ in $\mathscr{V}_{\varepsilon, \eta}$. Proof. Multiply (1) by $u_{x x x x t}+u_{x x x x}$; then we obtain

$$
\begin{aligned}
\frac{d}{d t}\left\{\frac{\eta}{2}\left\|u_{t x x}\right\|^{2}+\frac{\varepsilon^{2}}{2}\left\|u_{x x x x}\right\|^{2}\right. \\
\left.+\eta\left\langle u_{t x x}, u_{x x}\right\rangle+\frac{1}{2}\left\|u_{x x x}\right\|^{2}\right\} \\
+(1-\eta)\left\|u_{x x t}\right\|+\varepsilon^{2}\left\|u_{x x x x}\right\|^{2} \\
=\frac{1}{2}\left\langle\left[W^{\prime}\left(u_{x}\right)\right]_{x}, u_{x x x x t}+u_{x x x x}\right\rangle .
\end{aligned}
$$


We call $\theta_{0}(t)$ the differential term of the previous inequality and we estimate the r.h.s.:

$$
\begin{aligned}
\frac{1}{2}\left\langle\left[W^{\prime}\left(u_{x}\right)\right]_{x}, u_{x x x x}\right\rangle \\
\quad=-\frac{1}{2}\left\langle\left[W^{\prime}\left(u_{x}\right)\right]_{x x}, u_{x x x}\right\rangle \\
\quad=-\frac{1}{2}\left\langle W^{\prime \prime \prime}\left(u_{x}\right) u_{x x}^{2}, u_{x x x}\right\rangle-\frac{1}{2}\left\langle\left[W^{\prime \prime}\left(u_{x}\right)\right]_{x x}, u_{x x x}^{2}\right\rangle \\
\quad=-12 \int_{I} u_{x} u_{x x}^{2} u_{x x x} d x-2 \int_{I}\left(3 u_{x}^{2}-1\right) u_{x x x}^{2} d x \\
\quad \leq 2\left\|u_{x x x}\right\|^{2}+12\left\|u_{x x}\right\|_{\infty}^{2}\left\|u_{x}\right\|\left\|u_{x x x}\right\| \\
\quad \leq 2\left\|u_{x x x}\right\|^{2}\left(6\left\|u_{x x}\right\|^{2}+1\right) \\
\quad \leq \varepsilon^{2} R_{3}^{2}\left(\frac{12}{\varepsilon^{2}} R_{2}^{2}+1\right):=A_{1} .
\end{aligned}
$$

Let us define

$$
\theta_{1}(t):=\frac{1}{4} \int_{I} W^{\prime \prime}\left(u_{x}\right) u_{x x x}^{2} d x
$$

then

$$
\begin{aligned}
\frac{1}{2}\left\langle\left[W^{\prime}\left(u_{x}\right)\right]_{x}, u_{x x x x t}\right\rangle \\
=-\frac{1}{2}\left\langle\left[W^{\prime}\left(u_{x}\right)\right]_{x x}, u_{x x x t}\right\rangle \\
=-\frac{1}{2}\left\langle W^{\prime \prime \prime}\left(u_{x}\right) u_{x x}^{2}, u_{x x x t}\right\rangle \\
-\frac{1}{2}\left\langle W^{\prime \prime}\left(u_{x}\right) u_{x x x}, u_{x x x t}\right\rangle \\
=-\frac{d}{d t} \theta_{1}+\frac{1}{2}\left\langle\left[W^{\prime \prime \prime}\left(u_{x}\right) u_{x x}^{2}\right]_{x}, u_{x x t}\right\rangle \\
+\frac{1}{4} \int_{I} W^{\prime \prime \prime}\left(u_{x}\right) u_{x t} u_{x x x}^{2} d x .
\end{aligned}
$$

We estimate the last two terms of the previous equality:

$$
\begin{aligned}
& \frac{1}{4} \int_{I} W^{\prime \prime \prime}\left(u_{x}\right) u_{x t} u_{x x x}^{2} d x \\
& \quad=6 \int_{I} u_{x} u_{x t} u_{x x x}^{2} d x \\
& \quad \leq 6\left\|u_{x x x}\right\|_{\infty}\left\|u_{x}\right\|_{\infty}\left\|u_{x t}\right\|\left\|u_{x x x}\right\| \\
& \quad \leq 6\left\|u_{x x x}\right\|^{3 / 2}\left\|u_{x x x x}\right\|^{1 / 2}\left\|u_{x t}\right\|\left\|u_{x x}\right\|^{2} \\
& \quad \leq\left(12 \cdot 2^{3 / 4} \frac{R_{2}^{2} R_{3}^{3 / 2}}{\varepsilon^{7 / 2}}\right)\left\|u_{x x x x}\right\|^{1 / 2}\left\|u_{x t}\right\|
\end{aligned}
$$

$$
\begin{aligned}
& \leq\left(12 \cdot 2^{3 / 4} \frac{R_{2}^{2} R_{3}^{3 / 2}}{\varepsilon^{7 / 2}}\right)^{2}\left\|u_{x x x x}\right\|+\left\|u_{x t}\right\|^{2} \\
& \leq \frac{1}{2 \varepsilon^{2}}\left(12 \cdot 2^{3 / 4} \frac{R_{2}^{2} R_{3}^{3 / 2}}{\varepsilon^{7 / 2}}\right)^{4}+\frac{2}{\eta} R_{3}^{2}+\frac{\varepsilon^{2}}{2}\left\|u_{x x x x}\right\|^{2} \\
& :=\frac{\varepsilon^{2}}{2}\left\|u_{x x x x}\right\|^{2}+A_{2},
\end{aligned}
$$$$
\frac{1}{2}\left\langle\left[W^{\prime \prime \prime}\left(u_{x}\right) u_{x x}^{2}\right]_{x}, u_{x x t}\right\rangle
$$$$
=\frac{1}{2}\left\langle W^{i v}\left(u_{x}\right) u_{x x}^{3}+W^{\prime \prime \prime}\left(u_{x}\right) 2 u_{x x} u_{x x x}, u_{x x t}\right\rangle
$$$$
=12 \int_{I} u_{x x}^{3} u_{x x t} d x+24 \int_{I} u_{x} u_{x x} u_{x x x} u_{x x t} d x
$$$$
\leq 12\left(\left\|u_{x x}\right\|_{\infty}^{2}\left\|u_{x x}\right\|\right.
$$$$
\left.+2\left\|u_{x}\right\|_{\infty}\left\|u_{x x}\right\|_{\infty}\left\|u_{x x x}\right\|\right)\left\|u_{x x t}\right\|
$$$$
\leq 12\left(\left\|u_{x x}\right\|^{2}\left\|u_{x x x}\right\|\right.
$$$$
\left.+2\left\|u_{x x}\right\|^{3 / 2}\left\|u_{x x x}\right\|^{3 / 2}\right)\left\|u_{x x t}\right\|
$$$$
\leq \frac{\eta}{2}\left\|u_{x x t}\right\|^{2}+72\left(\left\|u_{x x}\right\|^{2}\left\|u_{x x x}\right\|\right.
$$$$
\left.+2\left\|u_{x x}\right\|^{3 / 2}\left\|u_{x x x}\right\|^{3 / 2}\right)^{2}
$$$$
\leq \frac{\eta}{2}\left\|u_{x x t}\right\|^{2}+72\left[\frac{2 \sqrt{2} R_{2}^{2} R_{3}}{\varepsilon^{3}}+2\left(\frac{2 R_{2} R_{3}}{\varepsilon^{2}}\right)^{3 / 2}\right]^{2}
$$$$
:=\frac{\eta}{2}\left\|u_{x x t}\right\|^{2}+A_{3} \text {. }
$$

Then if we set $\theta(t)=\theta_{0}(t)+\theta_{1}(t)$ we get

$$
\frac{d}{d t} \theta(t)+\left\|\left(u, u_{t}\right)\right\|_{\mathscr{V}_{\varepsilon, \eta}}^{2} \leq A_{1}+A_{2}+A_{3}:=A
$$

$$
\begin{aligned}
\theta(t) & \geq\left\|\left(u, u_{t}\right)\right\|_{\mathscr{V}_{\varepsilon, \eta}}^{2}-\frac{\eta}{4}\left\|u_{x x t}\right\|^{2}-\frac{1}{2}\left\|u_{x x}\right\|^{2} \\
& \geq \frac{1}{2}\left\|\left(u, u_{t}\right)\right\|_{\mathscr{V}_{\varepsilon, \eta}}^{2}-\frac{1}{2} R_{2}^{2} \\
& :=\frac{1}{2}\left\|\left(u, u_{t}\right)\right\|_{\mathscr{V}_{\varepsilon, \eta}}^{2}-a_{1},
\end{aligned}
$$

\section{Moreover we have}




$$
\begin{aligned}
\theta(t) \leq & \left\|\left(u, u_{t}\right)\right\|_{\mathscr{V}_{\varepsilon, \eta}}^{2}+\eta\left\|u_{x x t}\right\|\left\|u_{x x}\right\| \\
& +\frac{1}{2}\left\|u_{x x}\right\|^{2}+\frac{1}{4}\left\|W^{\prime \prime}\left(u_{x}\right)\right\|_{\infty}\left\|u_{x x x}\right\|^{3} \\
\leq & 2\left\|\left(u, u_{t}\right)\right\|_{\mathscr{V}_{\varepsilon, \eta}}^{2}+\left\|u_{x x}\right\|^{2} \\
& +\left(3\left\|u_{x}\right\|_{\infty}^{2}+1\right)\left\|u_{x x x}\right\|^{2} \\
\leq & 2\left\|\left(u, u_{t}\right)\right\|_{\mathscr{V}_{\varepsilon, \eta}}^{2}+\frac{2}{\varepsilon^{2}} R_{2}^{2}+\left(\frac{6}{\varepsilon^{2}} R_{2}^{2}+1\right) \frac{2}{\varepsilon^{2}} R_{3}^{2} \\
:= & 2\left\|\left(u, u_{t}\right)\right\|_{\mathscr{V}_{\varepsilon, \eta}}^{2}+a_{2} .
\end{aligned}
$$

Then putting all together we get

$$
\frac{1}{2}\left\|\left(u, u_{t}\right)\right\|_{\mathscr{V}_{\varepsilon, \eta}}^{2}-a_{1} \leq \theta(t) \leq 2\left\|\left(u, u_{t}\right)\right\|_{\mathscr{V}_{\varepsilon, \eta}}^{2}+a_{2} .
$$

From the previous inequality we get

$$
\frac{d}{d t} \theta(t)+\frac{1}{2} \theta(t) \leq A+\frac{a_{2}}{2}:=\widetilde{A}
$$

and by Gronwall's lemma

$$
\theta(t) \leq[\theta(0)-2 \widetilde{A}] e^{-(1 / 2) t}+2 \widetilde{A} .
$$

Using again (86) we get

$$
\begin{aligned}
\left\|\left(u, u_{t}\right)\right\|_{\mathscr{V}_{\varepsilon, \eta}}^{2} \leq & 2\left[2\left\|\left(u_{0}, u_{1}\right)\right\|_{\mathscr{V}_{\varepsilon, \eta}}^{2}+a_{2}-2 \widetilde{A}\right] e^{-(1 / 2) t} \\
& +4 \widetilde{A}+2 a_{1} .
\end{aligned}
$$

Then if $\left(u_{0}, u_{1}\right) \in \mathscr{V}_{\varepsilon, \eta}$ such that

$$
\left\|\left(u_{0}, u_{1}\right)\right\|_{\mathscr{V}_{\varepsilon, \eta}} \leq R
$$

then there exists $R_{4}>0$ :

$$
\left\|\left(u, u_{t}\right)\right\|_{\mathscr{V}_{\varepsilon, \eta}} \leq R_{4}, \quad \forall t \geq \tau_{V},
$$

where

$$
\tau_{V}:=2 \log \left[\frac{2\left(2 R^{2}+a_{2}-2 \widetilde{A}\right)}{R_{4}^{2}-4 \widetilde{A}-2 a_{1}}\right],
$$

and this concludes the proof.

\section{Global Attractor}

In this section we will show the existence of a global attractor for the semigroup $S_{\varepsilon, \eta}(t)$ in $\mathscr{H}_{\varepsilon, \eta}$. Since we have already proved the existence of the absorbing set in $\mathscr{H}_{\varepsilon, \eta}$, then it is sufficient (see, e.g., [4] or [14] for general results or [15] for a recent application on a weakly damped wave equation) to prove that, for any fixed bounded set $\mathscr{B} \subset \mathscr{H}_{\varepsilon, \eta}$, the solution semigroup $S_{\varepsilon, \eta}(t)$ admits the decomposition:

$$
S_{\varepsilon, \eta}(t)=L_{\varepsilon, \eta}(t)+N_{\varepsilon, \eta}(t),
$$

such that

$$
\begin{gathered}
\text { (C1) } \sup _{t \geq 0} \sup _{z \in \mathscr{H}_{\varepsilon, \eta}}\left\|L_{\varepsilon, \eta}(t) z\right\|_{\mathscr{V}_{\varepsilon, \eta}}<\infty, \\
\text { (C2) } \lim _{t \rightarrow \infty}\left\{\sup _{z \in \mathscr{H}_{\varepsilon, \eta}}\left\|N_{\varepsilon, \eta}(t) z\right\|_{\mathscr{H}_{\varepsilon, \eta}}\right\}=0 .
\end{gathered}
$$

Let $\mathscr{B} \subset \mathscr{H}_{\varepsilon, \eta}$ be a fixed bounded set and let $\left(u_{0}, u_{1}\right) \in$ $\mathscr{B} \subset \mathscr{H}_{\varepsilon, \eta}$. We will define the decomposition of $S_{\varepsilon, \eta}$ as follows:

$$
\begin{aligned}
& S_{\varepsilon, \eta}(t)\left(u_{0}, u_{1}\right)=\left(u, u_{t}\right), \\
& L_{\varepsilon, \eta}(t)\left(u_{0}, u_{1}\right)=\left(h, h_{t}\right), \\
& N_{\varepsilon, \eta}(t)\left(u_{0}, u_{1}\right)=\left(v, v_{t}\right),
\end{aligned}
$$

where $h$ and $v$ are solutions of the following problems:

$$
\begin{gathered}
\eta h_{t t}+h_{t}+\varepsilon^{2} h_{x x x x}-\alpha h_{x x} \\
=\frac{1}{2} W^{\prime \prime}\left(h_{x}\right) h_{x x}-\alpha u_{x x}, \\
h(x, 0)=0, \\
h_{t}(x, 0)=0, \\
h=h_{x x}=0, \quad \text { in } \partial I, \\
\eta v_{t t}+v_{t}+\varepsilon^{2} v_{x x x x}-\alpha v_{x x} \\
=\frac{1}{2} W^{\prime \prime}\left(u_{x}\right) u_{x x}-\frac{1}{2} W^{\prime \prime}\left(h_{x}\right) h_{x x}, \\
v(0)=u_{0}, \\
v_{t}(0)=u_{1}, \\
v=v_{x x}=0, \quad \text { in } \partial I,
\end{gathered}
$$

where

$$
\alpha \geq \frac{6 \sqrt{2}}{\varepsilon} R_{2}>6 \sqrt{6} .
$$

Before showing that the semigroups $L_{\varepsilon, \eta}(t)$ and $N_{\varepsilon, \eta}(t)$ satisfy the conditions (94) and (95), respectively, we consider the following lemma (see [9]) that will be useful for the sequel.

Lemma 10. Let $\psi: \mathbb{R}^{+} \rightarrow \mathbb{R}$ be an absolutely continuous function which fulfills, for some $v>0$ and almost every $t \geq 0$, the differential inequality

$$
\frac{d}{d t} \psi(t)+2 \nu \psi(t) \leq f(t) \psi(t),
$$

where $f$ is a positive function satisfying

$$
\int_{0}^{t} f(y) d y \leq v t+c, \quad \forall t \geq 0 .
$$

Then

$$
\psi(t) \leq c \psi(0) e^{-v t}, \quad t \geq 0 .
$$


5.1. The Semigroup $L_{\varepsilon, \eta}(t)$. Due to the similarity of problem (97) to (1), Proposition 9 still holds in this setting. We will omit the proof. Consequently we get that condition (94) holds by noting that $\left(h(0), h_{t}(0)\right)=(0,0)$.

Moreover by (97), multiplying the equation by $h_{t}$ and integrating over $I$, we get that there exists a constant $c>0$ such that

$$
\begin{aligned}
\frac{d}{d t}\left\{\frac{\eta}{2}\left\|h_{t}\right\|^{2}+\frac{\varepsilon^{2}}{2}\left\|h_{x x}\right\|^{2}\right. \\
\left.\quad+\frac{1}{2} \int_{I} W\left(h_{x}\right) d x+\frac{\alpha}{2}\left\|h_{x}\right\|^{2}+\alpha\left\langle u_{x x}, h\right\rangle\right\} \\
+\left\|h_{t}\right\|^{2}=\alpha\left\langle u_{t}, h_{x x}\right\rangle \leq c\left\|u_{t}\right\| .
\end{aligned}
$$

Then integrating the above equation on $(0, t)$ and using (30) we get that there exist constants $A>0$ and $B>0$ such that

$$
\begin{aligned}
\int_{0}^{t}\left\|h_{t}\right\|^{2} d s & \leq A+c \int_{0}^{t}\left\|u_{t}\right\| d s \\
& \leq A+B t^{1 / 2}
\end{aligned}
$$

Consequently, for any $\gamma>0$ there exists a constant $C_{\gamma} \geq 0$ such that

$$
\int_{0}^{t}\left\|h_{t}\right\|^{2} d s \leq \gamma \quad t+C_{\gamma}
$$

5.2. The Semigroup $N_{\varepsilon, \eta}(t)$. Let $\beta>0$ be a parameter to be determined later and let us multiply, in $L^{2}(I),(98)$ by $2 v_{t}+\beta v$. Then we get

$$
\begin{aligned}
\frac{d}{d t}\left\{\eta\left\|v_{t}\right\|^{2}+\eta \beta\left\langle v_{t}, v\right\rangle+\varepsilon^{2}\left\|v_{x x}\right\|^{2}\right. \\
+\frac{\beta}{2}\|v\|^{2}+\alpha\left\|v_{x}\right\|^{2} \\
\left.+\left\langle W\left(u_{x}\right)-W\left(h_{x}\right)-W^{\prime}\left(h_{x}\right) v_{x}, 1\right\rangle\right\} \\
+(2-\eta \beta)\left\|v_{t}\right\|^{2}+\varepsilon^{2} \beta\left\|v_{x x}\right\|^{2} \\
\quad-\frac{\beta}{2}\left\langle W^{\prime}\left(u_{x}\right)-W^{\prime}\left(h_{x}\right), v_{x}\right\rangle \\
=\left\langle h_{t x}, W^{\prime}\left(u_{x}\right)-W^{\prime}\left(h_{x}\right)-W^{\prime \prime}\left(h_{x}\right) v_{x}\right\rangle .
\end{aligned}
$$

We estimate the term involving $W$ and its derivatives:

$$
\begin{gathered}
-\frac{\beta}{2}\left\langle W^{\prime}\left(u_{x}\right)-W^{\prime}\left(h_{x}\right), v_{x}\right\rangle \\
=-\frac{\beta}{2}\left\langle W^{\prime \prime}\left(k_{x}\right) v_{x}, v_{x}\right\rangle \\
=2 \beta \int_{I}\left(1-3 k_{x}^{2}\right) v_{x}^{2} d x \\
\geq-6 \beta\left\|v_{x}\right\|_{\infty}\left\|v_{x}\right\|^{2} \\
\geq-\frac{6 \beta \sqrt{2}}{\varepsilon} R_{2}\left\|v_{x}\right\|^{2},
\end{gathered}
$$

where $k_{x}(x)$ is between $h_{x}(x)$ and $u_{x}(x)$ and satisfies $\left\|k_{x}\right\|_{\infty} \leq$ $\left\|v_{x}\right\|_{\infty}$. Moreover we have

$$
\begin{aligned}
\left\langle W\left(u_{x}\right)-W\left(h_{x}\right)-W^{\prime}\left(h_{x}\right) v_{x}, 1\right\rangle \\
=\left\langle W^{\prime}\left(h_{x}+\widetilde{k}_{x}\right)-W^{\prime}\left(h_{x}\right), v_{x}\right\rangle \\
=\left\langle W^{\prime \prime}\left(s_{x}\right) \widetilde{k}_{x}, v_{x}\right\rangle \\
=12 \int_{I} s_{x}^{2} \widetilde{k}_{x} v_{x} d x-4 \int_{I} k_{x} v_{x} d x \\
\geq-4\left\|v_{x}\right\|^{2},
\end{aligned}
$$

where $\tilde{k}_{x}(x)$ is between 0 and $v_{x}(x), s_{x}(x)$ is between 0 and $\tilde{k}_{x}(x)$ and $\left\|s_{x}\right\|_{\infty} \leq\left\|\widetilde{k}_{x}\right\|_{\infty} \leq\left\|v_{x}\right\|_{\infty}$. Moreover we have used that $v_{x}^{2} \geq \widetilde{k}_{x} v_{x} \geq 0$.

We denote as $\mathscr{F}$ the differential term of (106) and prove that it induces a norm that is equivalent to that of $\mathscr{H}_{\varepsilon, \eta}$. Then from (99) and (107) we have that

$$
\begin{aligned}
\mathscr{F}(t) \geq & \eta\left\|v_{t}\right\|^{2}-\frac{\eta}{2}\left\|v_{t}\right\|^{2}-\frac{\beta}{2}\|v\|^{2} \\
& +\varepsilon^{2}\left\|v_{x x}\right\|^{2}+\alpha\left\|v_{x}\right\|^{2}-\frac{6 \beta \sqrt{2}}{\varepsilon} R_{2}\left\|v_{x}\right\|^{2} \\
\geq & \frac{\eta}{2}\left\|v_{t}\right\|^{2}+\varepsilon^{2}\left\|v_{x x}\right\|^{2} \geq\left\|\left(v, v_{t}\right)\right\|_{\mathscr{H}}^{2} .
\end{aligned}
$$

Moreover we have

$$
\begin{aligned}
\mathscr{F}(t) \leq & \eta\left\|v_{t}\right\|^{2}+\frac{\eta}{2}\left\|v_{t}\right\|^{2}+\frac{\beta}{2}\|v\|^{2} \\
& +\varepsilon^{2}\left\|v_{x x}\right\|^{2}+\frac{\beta}{2}\|v\|^{2} \\
& +\alpha\left\|v_{x}\right\|^{2}+\left\|W^{\prime \prime}\left(k_{x}\right)\right\|_{\infty}\left\|v_{x}\right\|^{2} \\
\leq & \frac{3}{2} \eta\left\|v_{t}\right\|^{2}+\beta\left\|v_{x x}\right\|^{2}+\varepsilon^{2}\left\|v_{x x}\right\|^{2} \\
& +\alpha\left\|v_{x x}\right\|^{2}+4\left(3\left\|u_{x}\right\|_{\infty}^{2}+1\right)\left\|v_{x x}\right\|^{2} \\
\leq & \frac{3}{2} \eta\left\|v_{t}\right\|^{2}+\left(\beta+\varepsilon^{2}+\alpha+\frac{12 \sqrt{2} R_{2}}{\varepsilon}+4\right)\left\|v_{x x}\right\|^{2} \\
:= & \frac{3}{2} \eta\left\|v_{t}\right\|^{2}+A \frac{\varepsilon^{2}}{2}\left\|v_{x x}\right\|^{2} \\
\leq & c\left\|\left(v, v_{t}\right)\right\|_{\mathscr{H}}^{2},
\end{aligned}
$$

where we have used the same argument as (107) and (108) and where

$$
\bar{A}=\frac{2}{\varepsilon^{2}}\left(\beta+\varepsilon^{2}+\alpha+\frac{12 \sqrt{2} R_{2}}{\varepsilon}+4\right), \quad c=\max \{3, \bar{A}\} .
$$

Then we have

$$
\left\|\left(v, v_{t}\right)\right\|_{\mathscr{H}_{\varepsilon, \eta}}^{2} \leq \mathscr{F}(t) \leq c\left\|\left(v, v_{t}\right)\right\|_{\mathscr{H}_{\varepsilon, \eta}}^{2} .
$$


We estimate the right hand side of (106):

$$
\begin{aligned}
\left\langle h_{t x},\right. & \left.W^{\prime}\left(u_{x}\right)-W^{\prime}\left(h_{x}\right)-W^{\prime \prime}\left(h_{x}\right) v_{x}\right\rangle \\
= & \left\langle h_{t}, W^{\prime \prime \prime}\left(h_{x}\right) h_{x x} v_{x}+\left(W^{\prime \prime}\left(h_{x}\right)-W^{\prime \prime}\left(u_{x}\right)\right) u_{x x}\right\rangle \\
= & \left\langle h_{t}, 24 h_{x} h_{x x} v_{x}+12\left(h_{x}^{2}-u_{x}^{2}\right) u_{x x}\right\rangle \\
= & \left\langle h_{t}, 12 h_{x} v_{x}\left(2 h_{x x}-u_{x x}\right)-12 v_{x} u_{x} u_{x x}\right\rangle \\
= & \left\langle h_{t}, 12 v_{x}\left[h_{x}\left(h_{x x}-v_{x x}\right)-u_{x} u_{x x}\right]\right\rangle \\
= & \left\langle h_{t}, 12 v_{x}\left[-2 v_{x x} h_{x}-v_{x} u_{x x}\right]\right\rangle \\
\leq & 24\left\|h_{t}\right\|\left(\int_{I} v_{x}^{2} v_{x x}^{2} h_{x}^{2}\right)^{1 / 2} \\
& +12\left\|h_{t}\right\|\left(\int_{I} u_{x x}^{2} v_{x}^{4}\right)^{1 / 2} \\
\leq & 24\left\|h_{t}\right\|\left\|v_{x}\right\|_{\infty}\left\|h_{x}\right\|_{\infty}\left\|v_{x x}\right\| \\
& +12\left\|h_{t}\right\|\left\|v_{x}\right\|_{\infty}\left\|u_{x x}\right\|\left\|_{\infty}\right\| v_{x} \| \\
\leq & 12\left\|h_{t}\right\|\left\|v_{x x}\right\|^{2}\left(2\left\|h_{x x}\right\|+\left\|u_{x x x}\right\|\right) \\
\leq & \sigma\left\|h_{t}\right\|\left\|v_{x x}\right\|^{2} \\
\leq & \left\{\frac{2 \sigma^{2}}{\beta \varepsilon^{2}}\left\|h_{t}\right\|^{2}+\frac{\beta \varepsilon^{2}}{2}\right\}\left\|v_{x x}\right\|^{2},
\end{aligned}
$$

where

$$
\sigma=\frac{12 \sqrt{2}}{\varepsilon}\left(2 R_{2}+R_{3}\right) .
$$

Then it remains to estimate the following term:

$$
\begin{aligned}
& (2-\eta \beta)\left\|v_{t}\right\|^{2}+\beta \varepsilon^{2}\left\|v_{x x}\right\|^{2} \\
& +\alpha \beta\left\|v_{x}\right\|^{2}-\frac{\beta}{2}\left\langle W^{\prime}\left(u_{x}\right)-W^{\prime}\left(h_{x}\right), v_{x}\right\rangle \\
& \geq \beta \frac{\eta}{2}\left\|v_{t}\right\|^{2}+\beta \varepsilon^{2}\left\|v_{x x}\right\|^{2} \\
& +\alpha \beta\left\|v_{x}\right\|^{2}-6 \beta \frac{\sqrt{2}}{\varepsilon} R_{2}\left\|v_{x}\right\|^{2} \\
& \geq \beta \frac{\eta}{2}\left\|v_{t}\right\|^{2}+\beta \varepsilon^{2}\left\|v_{x x}\right\|^{2} .
\end{aligned}
$$

Then, using the above estimates, we can rewrite (106) in the following way:

$$
\begin{aligned}
\frac{d}{d t} \mathscr{F}(t)+\frac{\beta}{c} \mathscr{F}(t) & \leq \frac{\sigma^{2}}{2 \beta \varepsilon^{2}}\left\|h_{t}\right\|^{2}\left\|v_{x x}\right\|^{2} \\
& \leq \frac{\sigma^{2}}{\beta \varepsilon^{4}}\left\|h_{t}\right\|^{2}\left\|\left(v, v_{t}\right)\right\|_{\mathscr{H}_{\varepsilon, \eta}}^{2} \\
& \leq \frac{\sigma^{2}}{\beta \varepsilon^{4}}\left\|h_{t}\right\|^{2} \mathscr{F}(t) .
\end{aligned}
$$

Then by Lemma 10 and from (105) we conclude that there exists a positive constant $K$ such that

$$
\mathscr{F}(t) \leq K \mathscr{F}(0) e^{-(\beta / 2 c) t},
$$

and consequently from (112) we conclude

$$
\left\|\left(u, u_{t}\right)\right\|_{\mathscr{H}_{\eta, \varepsilon}}^{2} \leq c K\left\|\left(u_{0}, u_{1}\right)\right\|_{\mathscr{H}_{\eta, \varepsilon}}^{2} e^{-(\beta / 2 c) t} .
$$

This shows that the semigroup $N_{\varepsilon, \eta}(t)$ satisfies condition (95) and hence we have proven the following.

Theorem 11. The semigroup $S_{\varepsilon, \eta}(t)$ possesses a connected global attractor $\mathscr{A}_{\varepsilon, \eta} \subset \mathscr{H}_{\varepsilon, \eta}$ that is bounded in $\mathscr{V}_{\varepsilon, \eta}$.

\section{Acknowledgment}

The authors would like to thank the editor and the referees for their helpful suggestions and remarks. This work was partially supported by projects PPTA 4476 and PPTA 5571 of Pontificia Universidad Javeriana, Bogotá.

\section{References}

[1] G. Bellettini, G. Fusco, and N. Guglielmi, "A concept of solution and numerical experiments for forward-backward diffusion equations," Discrete and Continuous Dynamical Systems. Series A, vol. 16, no. 4, pp. 783-842, 2006.

[2] S. Müller, "Variational models for microstructure and phase transitions," in Calculus of Variations and Geometric Evolution Problems (Cetraro, 1996), vol. 1713 of Lecture Notes in Mathematics, pp. 85-210, Springer, Berlin, Germany, 1999.

[3] R. Colucci and G. R. Chacón, "Asymptoticbehavior of a fourth order evolution equation," Submitted paper.

[4] R. Temam, Infinite-Dimensional Dynamical Systems in Mechanics and Physics, vol. 68 of Applied Mathematical Sciences, Springer, New York, NY, USA, 2nd edition, 1997.

[5] R. Colucci and G. R. Chacón, "Dimension estimate for the global attractor of an evolution equation," Abstract and Applied Analysis, vol. 2012, Article ID 541426, 18 pages, 2012.

[6] P. Constantin, C. Foias, B. Nicolaenko, and R. Temam, Integral Manifolds and Inertial Manifolds for Dissipative Partial Differential Equations, vol. 70 of Applied Mathematical Sciences, Springer, New York, NY, USA, 1st edition, 1988.

[7] J. C. Robinson, Infinite-Dimensional Dynamical Systems: An Introduction to Dissipative Parabolic PDEs and the Theory of Global Attractors, Cambridge Texts in Applied Mathematics, Cambridge University Press, Cambridge, Mass, USA, 2001.

[8] P. Galenko, "Phase field model with relaxation of the diffusion flux in non equilibrium solidification of a binary system," Physiscs Letters A, vol. 287, pp. 190-197, 2001.

[9] S. Gatti, M. Grasselli, A. Miranville, and V. Pata, "On the hyperbolic relaxation of the one-dimensional Cahn-Hilliard equation," Journal of Mathematical Analysis and Applications, vol. 312, no. 1, pp. 230-247, 2005.

[10] S. Zheng and A. Milani, "Global attractors for singular perturbations of the Cahn-Hilliard equations," Journal of Differential Equations, vol. 209, no. 1, pp. 101-139, 2005.

[11] A. Segatti, "On the hyperbolic relaxation of the Cahn-Hilliard equation in 3D: approximation and long time behaviour," Mathematical Models \& Methods in Applied Sciences, vol. 17, no. 3, pp. 411-437, 2007. 
[12] M. Grasselli, G. Schimperna, A. Segatti, and S. Zelik, "On the 3D Cahn-Hilliard equation with inertial term," Journal of Evolution Equations, vol. 9, no. 2, pp. 371-404, 2009.

[13] H. Brezis, Functional Analysis, Sobolev Spaces and Partial Differential Equations, Universitext, Springer, New York, NY, USA, 2011.

[14] J. K. Hale, Asymptotic Behavior of Dissipative Systems, vol. 25 of Mathematical Surveys and Monographs, American Mathematical Society, Providence, RI, USA, 1988.

[15] S. Gatti and V. Pata, "A one-dimensional wave equation with nonlinear damping," Glasgow Mathematical Journal, vol. 48, no. 3, pp. 419-430, 2006. 


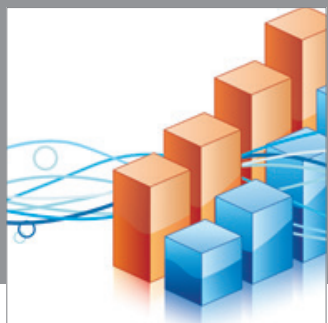

Advances in

Operations Research

mansans

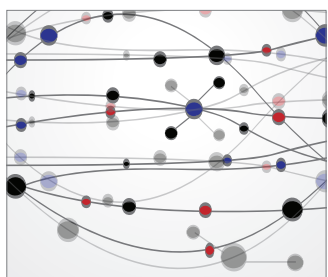

The Scientific World Journal
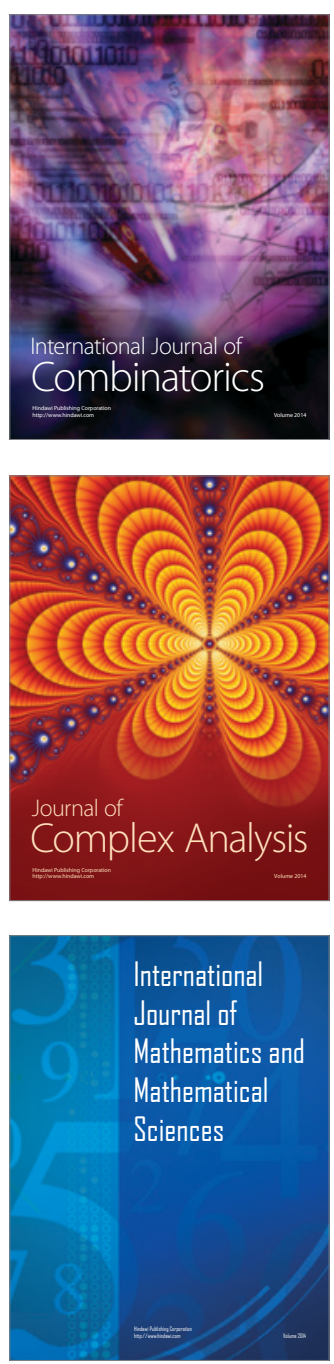
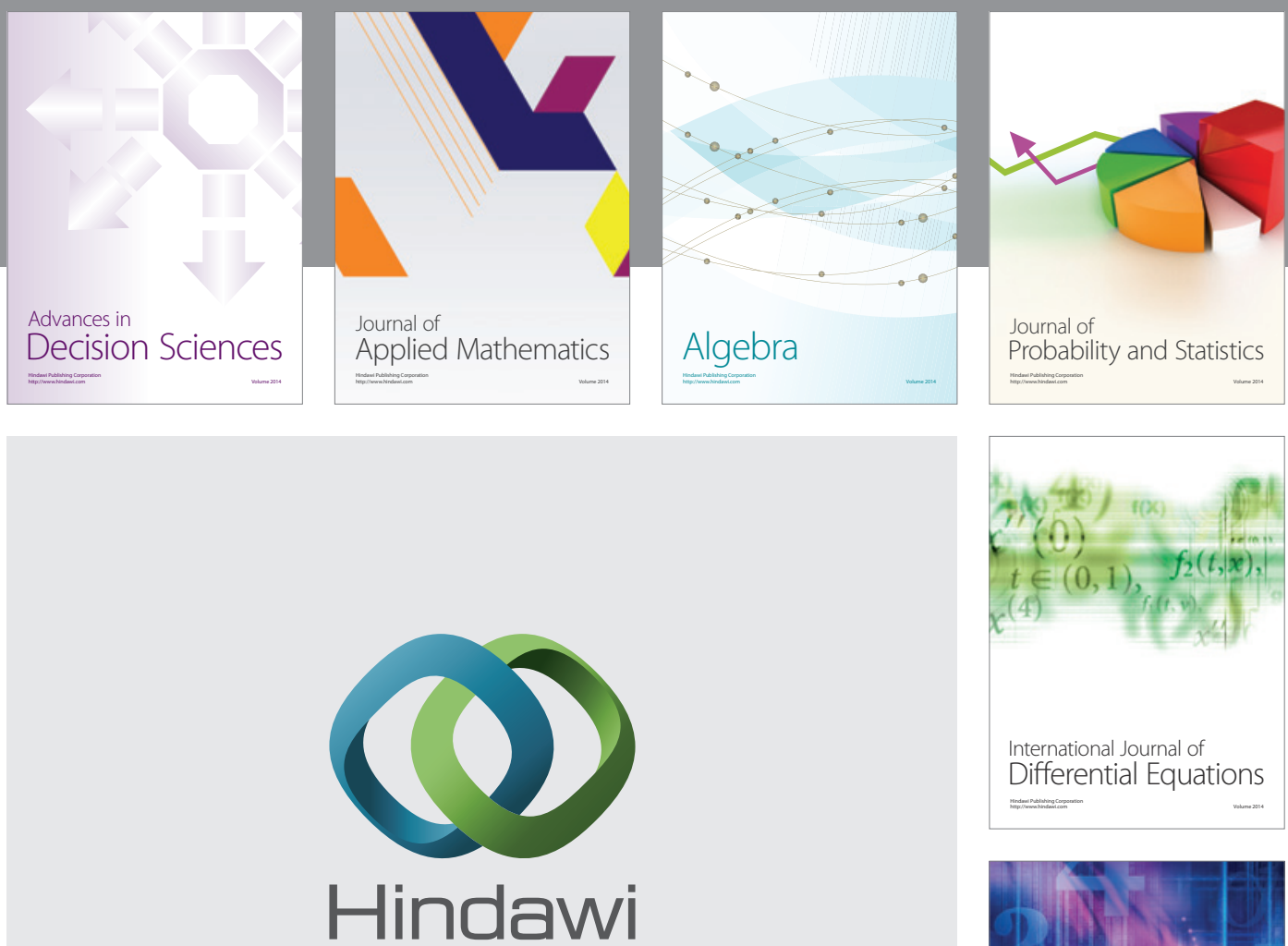

Submit your manuscripts at http://www.hindawi.com
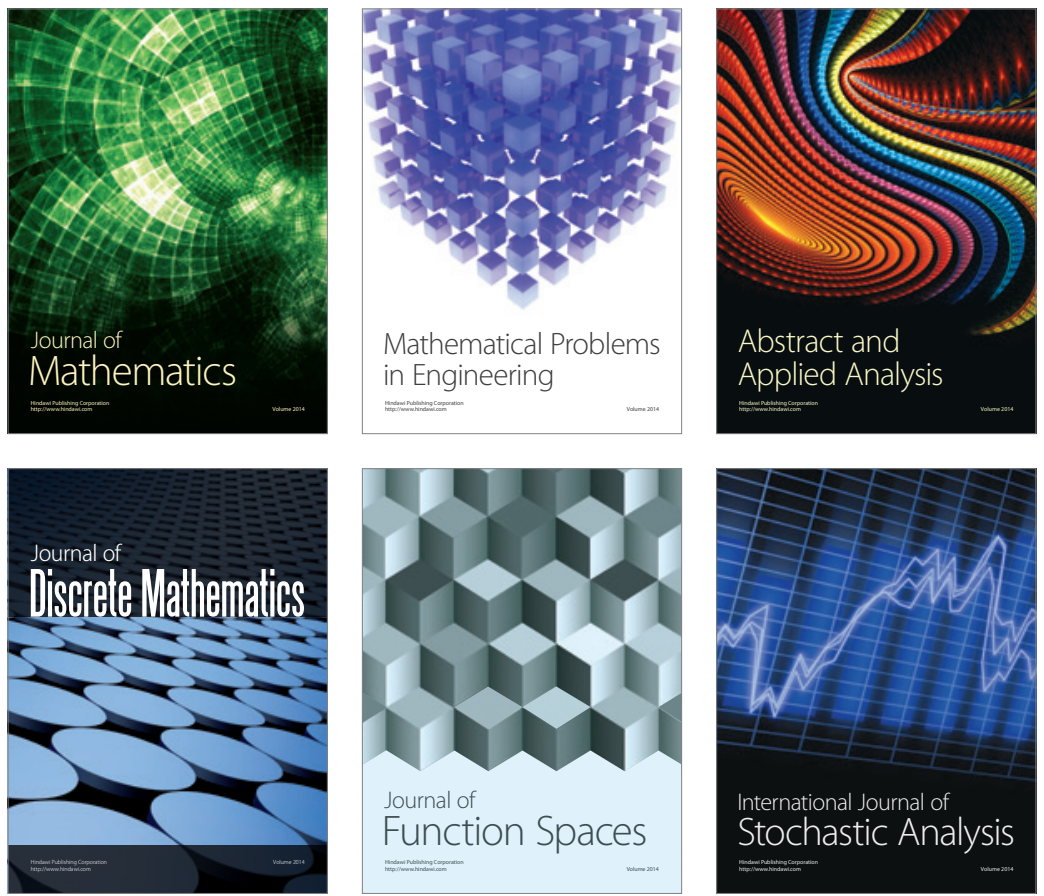

Journal of

Function Spaces

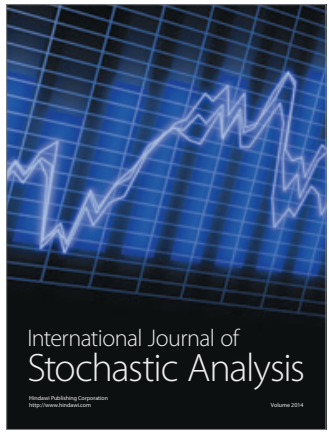

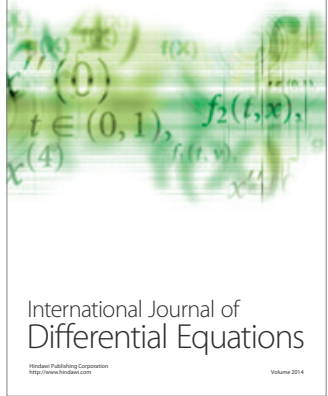
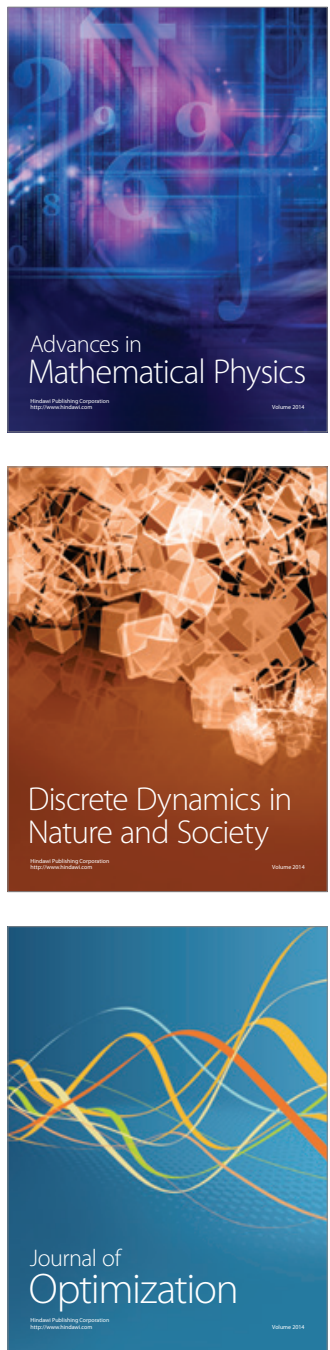